\title{
Terrestrial and lunar gravitational forces upon the mass of a cell: relevance to cell function
}

\author{
Michael A. Persinger \\ Laurentian University, 935 Ramsey Lake Road, Sudbury, Ontario P3E 2C6, Canada \\ E-mail address: mpersinger@laurentianl.ca
}

\begin{abstract}
The gravitational force between the mass of an average mammalian cell and the earth is in the order of a picoNewton. When applied across the plasma membrane the energy is within the domain of $10^{-20} \mathrm{~J}$ that is associated with the distance between forces of ions that are correlated with the membrane potential. Solutions for velocity and acceleration are congruent with known properties of the ion channel and cell membrane. The differences in gravitational forces between lunar perigee and apogee are within the $10^{-20} \mathrm{~J}$ range when applied across distances that constitute neuronal processes. Calculations of the ratio of gravitational force to a specific range of intensities of rotating experimental magnetic fields produce equivalent electric dipole moments (A.m) that are within the same order of magnitude as that measured for single post-synaptic potentials. These values match the energies associated with pressures within the cell volume according to Borowski's gravitational theory. The solutions may explain a robust behavioral effect reported over 40 years ago that indicated a powerful interaction between forces associated with lunar distances at the birth of rats exposed prenatally to rotating magnetic fields. Although the energies may be small the contributions from subtle changes in gravitational forces are within the operative range for those that influence cell function.
\end{abstract}

Keywords: gravitational effects; cell mass; perigee-apogee effects; $10^{-20} \mathrm{~J}$ quantum; magnetic fieldlunar distance interactions; postsynaptic potentials; electric dipole moment

\section{INTRODUCTION}

The masses of the earth $\left(5.98 \cdot 10^{24} \mathrm{~kg}\right)$ and the moon $\left(7.34 \cdot 10^{22} \mathrm{~kg}\right)$ compared to the mass of a single mammalian cell $\left(\sim 10^{-13} \mathrm{~kg}\right)$ would seem extraordinarily too disproportional to be related. Although the gravitational force between these two major bodies is known to produce tidal forces that affect the height of oceans as a dynamic (rotational) process, the gravitational forces between the mass of cells in organisms and the earth have been assumed to be too minimal to be relevant. Yet intrinsic $\sim 28$ day periodicities in many living systems have been documented and may be even emerge following brain injury [1]. That lunar phase affects the movement of different classes of vertebrates which can be modified by applied weak $\left(\sim 10^{-4} \mathrm{~T}\right)$ horizontal static magnetic fields was elegantly explored by Brown [2,3].

If Life evolved on the surface of the earth within a more or less constant gravitational context, there should be compatible quantities of energy derived from this force that may set its physical and chemical boundaries. Given the emergence of new approaches to gravitation [4], a re-evaluation of this universal force with respect to its biological implications may be 
revealing. Previous calculations [5] have indicated quantitative similarities between electromagnetic and gravitational phenomena. Here I present quantitative evidence that the gravitational energy between the mass of a single cell and the earth is within the order of magnitude of the energy that maintains the resting plasma membrane potential and that very weak lunar energies that change between perigee and apogee may be more important than anticipated for emergent neurophysiological functions.

\section{GRAVITATIONAL EFFECTS UPON THE AVERAGE CELL}

The mass of a single mammalian cell with a diameter of $10 \mu \mathrm{m}$ and the density of water is $5.23 \cdot 10^{-13} \mathrm{~kg}$. If specific density is accommodated the actual value would be multiplied by $\sim 1.09$. Here I am assuming the average cell soma is $10 \mu \mathrm{m}$ in diameter for two reasons. First, it is the approximate solution from Wien's law of $0.29 \mathrm{~cm} \cdot T \cdot \lambda^{-1}$; the wavelength for a body at $310^{\circ} \mathrm{K}\left(37^{\circ} \mathrm{C}\right)$ is $\sim 10 \mu \mathrm{m}$. Secondly, the energy equivalent for a frequency with this wavelength moving at $\mathrm{c}$, the velocity of light in a vacuum, is around $10^{-20} \mathrm{~J}$ which has been shown to be a fundamental unit [6].

The product of the mass of the earth and of a cell divided by the square of the distance of the earth's radius $\left(6.38 \cdot 10^{6} \mathrm{~m}^{2}\right)$ when multiplied by the gravitational constant $\mathrm{G}\left(6.624 \cdot 10^{-}\right.$ $\left.{ }^{11} \mathrm{~m}^{3} \cdot \mathrm{kg}^{-1} \cdot \mathrm{s}^{-2}\right)$ is about $5.1 \cdot 10^{-12} \mathrm{~N}$. If the force is applied across the width of a membrane $\left(10^{-8}\right.$ $\mathrm{m})$ the energy is $\sim 10^{-20} \mathrm{~J}$. This quantum of energy is associated with the action potential $(\Delta \mathrm{V}$ $\left.=1.2 \cdot 10^{-1} \mathrm{~V} \cdot 1.6 \cdot 10^{-19} \mathrm{~A} \cdot \mathrm{s}=1.9 \cdot 10^{-20} \mathrm{~J}\right)$, the energy from the electric force over the distance between the individual charges that generate the resting membrane potential, and the range of energies associated with a variety of biologically relevant components of chemical bonds.

The intrinsic velocity from this relationship $\left[\left(5.23 \cdot 10^{-13} \mathrm{~kg}\right) \cdot\left(6.6 \cdot 10^{-11} \mathrm{~m}^{3} \cdot \mathrm{kg}^{-1} \cdot \mathrm{s}^{-2}\right)\right]$ divided by the radius of the cell $\left(5 \cdot 10^{-6} \mathrm{~m}\right)$ is $6.9 \cdot 10^{-18} \mathrm{~m}^{2} \cdot \mathrm{s}^{-2}$ or $2.6 \cdot 10^{-9} \mathrm{~m} \cdot \mathrm{s}^{-1}$. This value is within the order of magnitude of the velocity of a $\mathrm{K}^{+}$ion moving through a membrane without open channels. When the channels are open and passive diffusion without resistance occurs, the velocity is in the order of $10^{-1} \mathrm{~m} \cdot \mathrm{s}^{-1}$.

The intrinsic acceleration associated with the average cell mass $\left[\left(5.23 \cdot 10^{-13} \mathrm{~kg}\right)\right.$. $\left.\left(6.6 \cdot 10^{-11} \mathrm{~m}^{3} \cdot \mathrm{kg}^{-1} \cdot \mathrm{s}^{-2}\right)\right]$ divided by the square area of the soma $\left(2.5 \cdot 10^{-11} \mathrm{~m}^{2}\right)$ is $1.38 \cdot 10^{-12} \mathrm{~m} \cdot \mathrm{s}^{-2}$. When this acceleration is applied over the mass of the cell the force would be $\sim 7.2 \cdot 10^{-25} \mathrm{~N}$. Although minute, if this is concentrated at the boundary condition, the membrane $\left(10^{-8} \mathrm{~m}\right)$ the energy would be $7.2 \cdot 10^{-33} \mathrm{~J}$. The intrinsic frequency associated with this gravitational energy would be, when divided by Plank's constant, $\sim 10 \mathrm{~Hz}$. Such vibrations are intrinsic properties for many cells, including those within the center of the thalamus (midline nuclei) that are the $\sim 10 \mathrm{~Hz}$ "pacemaker" cells that drive the characteristics of the alpha rhythm over the cerebral cortices [7].

The gravitational force between two cells is also significant. Assuming a cell radius of separation $(5 \mu \mathrm{m})$ between two somas, the force would be $7.2 \cdot 10^{-25} \mathrm{~N}$. When applied across the plasma membrane of $10 \mathrm{~nm}$, the energy would be $7.2 \cdot 10^{-33} \mathrm{~J}$. Here too, the intrinsic oscillatory component would be $\sim 10 \mathrm{~Hz}$.

\section{LUNAR FORCE VARIATIONS}

Assuming a mass of $5.23 \cdot 10^{-13} \mathrm{~kg}$ for a classic cell, the mass of the moon $\left(7.35 \cdot 10^{22} \mathrm{~kg}\right)$ and $\mathrm{G}$, the gravitational force on that cell at perigee $\left(3.633 \cdot 10^{8} \mathrm{~m}\right)$ would be $1.95 \cdot 10^{-17} \mathrm{~N}$. The 
gravitational force at apogee $\left(4.055 \cdot 10^{8} \mathrm{~m}\right)$ would be $1.57 \cdot 10^{-17} \mathrm{~N}$. Again, although apparently minuscule, if these forces were applied over the length of $1 \mathrm{~mm}\left(10^{-3} \mathrm{~m}\right)$, which would accommodate the order of magnitude of the developing rat brain, the energies would be in the order of $10^{-20} \mathrm{~J}$. This length is also within the range of space that comprises the aggregates of tens of thousands of neurons that define nuclei within the brain or ganglia in the peripheral nervous system.

If the mass of a neuron's soma with a diameter of $10 \mu \mathrm{m}$ and its processes (which accommodate about $90 \%$ of the surface area of the cell) are considered, the cellular mass would be $\sim 5.66 \cdot 10^{-12} \mathrm{~kg}$ and the gravitational force from lunar distance at perigee $\left(2.1 \cdot 10^{-16}\right.$ $\mathrm{N})$ and apogee $\left(1.69 \cdot 10^{-16} \mathrm{~N}\right)$ would be approximately an order of magnitude larger. When applied across the typical dendritic field of $0.1 \mathrm{~mm}(100 \mu \mathrm{m})$ the energies would be $2.10 \cdot 10^{-20}$ $\mathrm{J}$ and $1.69 \cdot 10^{-20} \mathrm{~J}$, respectively. This increment of energy has been considered a fundamental neuroquantal quantity that is associated with the neuronal action potential $\left(1.2 \cdot 10^{-1} \mathrm{~V}\right.$ peak-topeak multiplied by $1.6 \cdot 10^{-19} \mathrm{~A} \cdot \mathrm{s}$, or $1.9 \cdot 10^{-20} \mathrm{~J}$ ), the energy between the potassium ions associated with the resting membrane potential, and critical values for sequestering ligands to receptors [6].

These energies are correlated with the small quantities of chemicals that mediate the temporal patterns of information across the interface (the synapse) between surfaces of membranes. The postsynaptic shift in voltage from the release of the molecular contents within a single vesicle is about $0.5 \mathrm{mV}$ and defines a miniature EPSP (excitatory post synaptic potential) or ISP (inhibitory post synaptic potential). According to Kandel et al [8], a single acetylcholine receptor is associated with a shift of $0.3 \mu \mathrm{V}$. If the average current of a channel, $1 \mathrm{pA}\left(10^{-12} \mathrm{~A}\right)$, occurs, then the implicit resistance would be $\left(3 \cdot 10^{-6} \mathrm{~V}\right.$ divided by $\left.10^{-12} \mathrm{~A}\right)$ or $3 \cdot 10^{5} \Omega$. Capacitance is $\Omega^{-1} \cdot \mathrm{s}$. Consequently $\left(0.3 \cdot 10^{-5} \Omega^{-1}\right) \cdot\left(2 \cdot 10^{-2} \mathrm{~s}\right)$, the typical duration $(20$ $\mathrm{ms})$ of activity in dendritic processes [9], results in $0.6 \cdot 10^{-7}$ Farads. This product of the capacitance and the voltage is time, or $1.8 \cdot 10^{-14} \mathrm{~s}$. The quantum energy equivalence for this duration when divided into Planck's constant of $6.626 \cdot 10^{-34} \mathrm{~J} \cdot \mathrm{s}$ is $\sim 3 \cdot 10^{-20} \mathrm{~J}$.

In other words, the magnitude of energy beginning the processes that determine if a neuronal membrane will discharge or not is within the same order of magnitude as the energy associated with the gravitational force between the moon and the mass of an average neuron. That very, very small energies associated with relatively small forces can be disproportionately effective can be shown by demonstration. For example the force from dropping a small candy $\left(10^{-3} \mathrm{~kg}\right)$ from $0.5 \mathrm{~m}$ may only be in the order of milliNewtons with energy in the microJoule range. Given the threshold of the human dark adapted eye to the detection of light is $10^{-17} \mathrm{~J}$, this means that the energy from that small mechanical pressure if it were electromagnetic energy could produce a light experience in every person on the planet if they were dark adapted [10].

\section{BOROWSKI GRAVIATIONAL PRESSURE EQUIVALENTS}

Borowski's theory of gravitation [4,11] indicates that differential pressure in the solar system more appropriately describes the motion of mass-holding bodies. The concept of attraction, intrinsic to Newtonian metaphors, is less applicable. Application of this assumption for the gravitation force at perigee $\left(1.95 \cdot 10^{-17} \mathrm{~N}\right)$ to an average cell with an exposure area of $7.85 \cdot 10^{-11} \mathrm{~m}^{2}$ would result in a functional "pressure" of $0.25 \cdot 10^{-6} \mathrm{~Pa}$ or about 100 times below the threshold for hearing between $>100 \mathrm{~Hz}$ and $<5 \mathrm{kHz}$ acoustic oscillations [10]. 
Because the product of pressure and volume is energy, the resultant energy would be $0.25 \cdot 10^{-6} \mathrm{~Pa} \cdot 5.23 \cdot 10^{-16} \mathrm{~m}^{3}$ or $1.33 \cdot 10^{-22} \mathrm{~J}$. When applied to the unit charge $\left(1.6 \cdot 10^{-19} \mathrm{~A} \cdot \mathrm{s}\right)$ this quantum of energy would be equivalent to the presence of $0.8 \mathrm{mV}$. This is well within the range of the increment of energy associated with a miniature EPSP or IPSP at the synapse.

The rate of change of this pressure compliments the time-course of critical synaptic and membrane events. For example the net change in gravitational force from the moon upon the mass of a cell between perigee and apogee is $3.86 \cdot 10^{-18} \mathrm{~N}$ per $1.18 \cdot 10^{6} \mathrm{~s}(\sim 13.66$ days $)$ or $3.27 \cdot 10^{-24} \mathrm{~N} \cdot \mathrm{s}^{-1}$. When this value is divided by the area of the cell $\left(7.85 \cdot 10^{-11} \mathrm{~m}^{2}\right)$, this means that for every second there is a change of $0.42 \cdot 10^{-13} \mathrm{~Pa}$. Within the volume of the cell, the energy would be $2.2 \cdot 10^{-29} \mathrm{~J}$. The intrinsic quantum frequency associated with the value is $0.33 \cdot 10^{5} \mathrm{~Hz}$ or the time increments (the inverse) of $3 \cdot 10^{-5} \mathrm{~s}(30 \mu \mathrm{s})$. This is within error measurement for the time required for closure of pores within voltage sensitive domains for potassium in cell membranes [12] and the first phase of the biphasic peak from a pulse of neurotransmitter across the membrane [13].

If this same rate of change in dynamic pressure from the changing gravitational forces of the moon between apogee and perigee were applied across the cell $\left(10^{-5} \mathrm{~m}\right)$ the power would be $3.22 \cdot 10^{-29} \mathrm{~W}$. At such minute quantities where quantum energies become potentially relevant when applied to those spaces, such as the cross-sectional area of the Bohr magneton $\left(8.79 \cdot 10^{-19} \mathrm{~m}^{2}\right)$, the power density emerges as $\sim 3 \cdot 10^{-11} \mathrm{~W} \cdot \mathrm{m}^{-2}$. This is within the range of the radiant flux density of photon fields emitted from living cerebral tissue [14]. Recently Moraes et al [15] showed that spontaneous ultra-weak light emissions from wheat seedlings were synchronized with the gravimetric tide, more specifically the lunisolar tidal accelerations.

\section{A POTENTIAL APPLICATION TO EXPERIMENTAL RESULTS}

One of the most robust phenomena I have measured over the last 50 years has been the interaction between experimentally applied rotating magnetic fields during prenatal development in the rodent and the distance of the moon at the time of the litter's birth upon behavior. Litters were considered the unit rather than the individual because the numbers $(8<$ $\mathrm{n}<16$ ) of rat pups within a litter are born to the same source within durations of two to three hours. As can be seen in Figure 1, which reproduces the original scattergram from 1971 [16], the scalar value (number of squares traversed) of the open field ambulatory behavior of litters $(n=19)$ of rats upon weaning (25 days of age) that had been exposed during their entire prenatal development to a 29 RPM $(0.48 \mathrm{~Hz})$ rotating magnetic field with field strengths between $3 \cdot 10^{-4}$ and $3 \cdot 10^{-3} \mathrm{~T}$ displayed a very strong correlation $(\mathrm{r}=0.88)$ with the apogeeperigee distances of the moon at the times of their births. The correlation between lunar distance and post-weaning ambulation in control litters $(n=12)$ was not significant statistically $(\mathrm{r}=0.10)$. The experimental pregnant females had been exposed between two horseshoe magnets that were rotating in opposite directions. The litters were born over a one year period.

Because only those litters that had been exposed to the rotating magnetic fields displayed the perigee-apogee effect, an interaction between the gravitational forces and the magnetic field intensity would be a potential candidate to accommodate the effect. The most cogent solution of physiological relevance would be the gravitational force divided by the magnetic field strength which results in A.m or current dipole moment. For the gravitational force $\left(1.95 \cdot 10^{-17} \mathrm{~N}\right)$ on the cell mass at perigee within a $3 \cdot 10^{-3} \mathrm{~T}$, the equivalent current dipole (ECD) moment would be $\sim 6.5 \mathrm{fA} \cdot \mathrm{m}\left(10^{-15} \mathrm{~A} \cdot \mathrm{m}\right)$. The value at apogee would be $\sim 5.2 \mathrm{fA} \cdot \mathrm{m}$, or 
a difference of about $25 \%$. For neurons, whose soma contributes about $10 \%$ to the total surface area, and hence volume, the calculated ECD moment at perigee and apogee would be $70 \mathrm{fA} \cdot \mathrm{m}$ and $56 \mathrm{fA} \cdot \mathrm{m}$, respectively.

The solution of within the order of $10^{-15} \mathrm{~A} \mathrm{~m}$ range is physiologically relevant. A dendrite current can be considered a current dipole (compared to the quadruple for an axon) with a moment defined by $\mathrm{Q}=\mathrm{I}_{\mathrm{i}} \lambda$ where $\mathrm{I}_{\mathrm{i}}$ the intracellular current. In this context the intracellular current moves through the longitudinal axis of the dendrite (analogous to the A vector in magnetic potential); the volume current returns through the adjacent extracellular space. Here I assume, as did Hamalainen et al [17] that the length constant, $\lambda$, of the dendrite current is similar to that within the human neocortex which is on average $0.1 \mathrm{~mm}$ for the lower boundary. The resultant current available from the ECD moment from the interaction between the magnetic field and the gravitational field at perigee for the neuron would be $\sim 0.7$ $\mathrm{nA}$ while this value for apogee would be $\sim 0.56 \mathrm{nA}$.

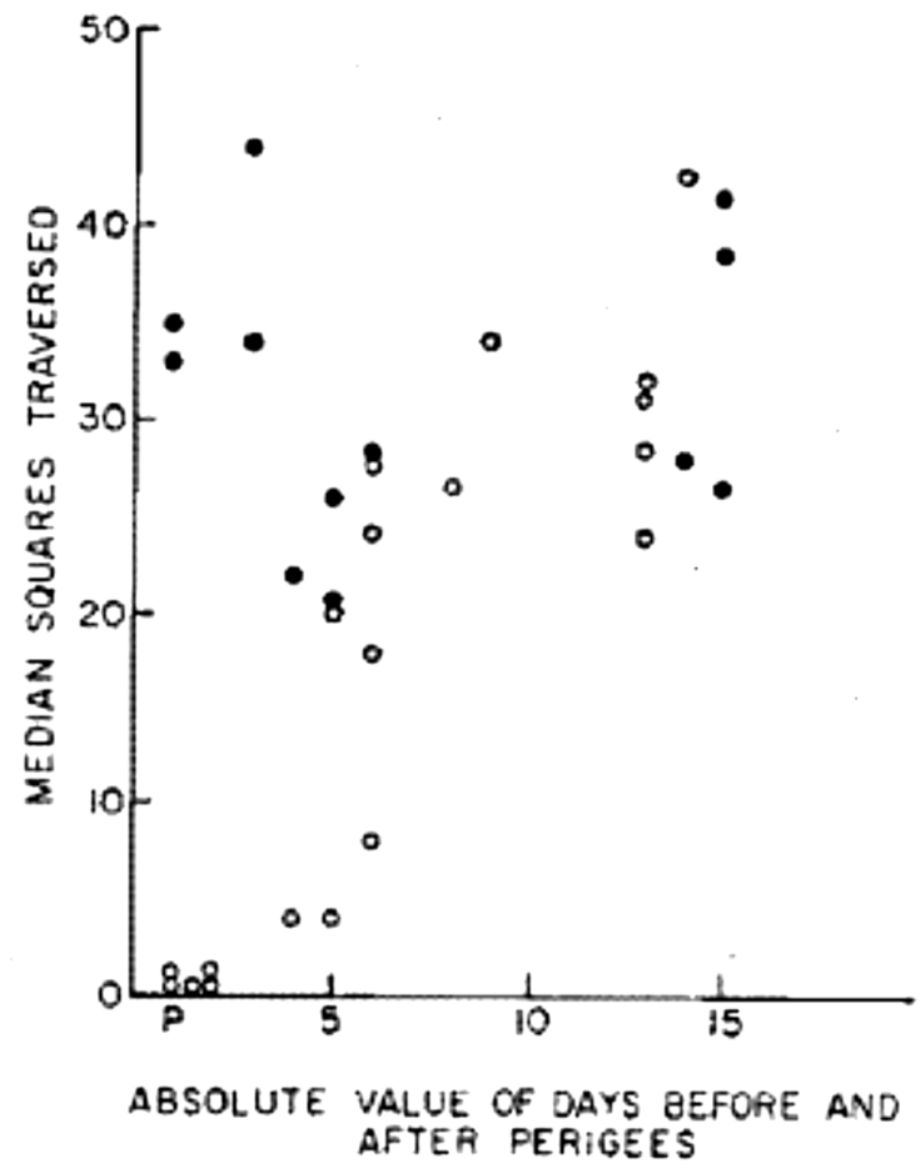

Figure 1. Median number of squares traversed (ambulation) for each rat litter at weaning as a function of the days before and after lunar perigee for groups exposed during their entire prenatal development to a $0.48 \mathrm{~Hz}, 3 \mathrm{mT}$ rotating magnetic field 3 (open circles) or to the sham-field (static geomagnetic field $\sim 50 \mu \mathrm{T}$ ) indicated by closed circles (from Persinger, 1971 [16]).

According to Park and Lee [18] the equivalent current dipole moment is in the order of $20 \cdot 10^{-15} \mathrm{~A} \cdot \mathrm{m}$ when produced by a single postsynaptic potential of $\sim 0.3 \mathrm{mV}$ [8]. The integrated postsynaptic current at the soma ranges between 1 and $5 \mathrm{nA}$ [18]. If one assumes the median 
of $2.5 \mathrm{nA}$, for this value, then the number of initial post synaptic currents would be 3.5 during perigee compared to 4.5 during apogee. In other words the ECD moment contribution from the interaction between gravitational forces at perigee and the upper boundary of the intensities of the applied magnetic field would require fewer indigenous currents to produce an integrated postsynaptic current in the soma because of the "continuous" contribution from the perigee-apogee effect. In addition the additional contribution from the ECD moment would encourage more postsynaptic activity which is important for the stability of neuronal networks. Because interneurons dominate the neuronal population and their functions are primarily inhibitory, the general enhancement would be associated with less ambulatory activity.

It may not be coincidence that the robust interaction between lunar distance at birth and exposure to magnetic fields during development involved a $0.48 \mathrm{~Hz}$ rotating magnetic field. Considering the slight slippage in the belts that controlled the rotating magnets the actual frequency may have been approaching 0.46 or $0.47 \mathrm{~Hz}$. Assuming c, the velocity of light in a vacuum, the intrinsic frequency of the width of the perigee diameter would be $0.41 \mathrm{~Hz}$ while that of apogee would be $0.37 \mathrm{~Hz}$. Even if the velocity were reduced to $2 \cdot 10^{8} \mathrm{~m} \cdot \mathrm{s}^{-1}$, and the values approached $0.5 \mathrm{~Hz}$, the frequency associated with the perigee distance would still, in the balance of probabilities, overlap more with that of the experimental field. Such similarity would be expected to promote resonance.

\section{CONCLUSIONS}

The role of gravitational forces and the corresponding magnitudes of energies during evolution upon the physical constraints of the cell and membrane function may be more important than traditionally considered. The emergence of the basic bioquantum unit of $10^{-20} \mathrm{~J}$ when the gravitational force between the masses of the earth and a typical cell was applied across the plasma cell membrane could reflect the origin of this biological boundary as well as a yet to be determine role of gravitational energy in cell function. This gravitational constraint upon biological systems because they emerged on this planet could significantly influence the adaptation of any terrestrial life forms during maintained planetary exploration or maintained presence on worlds with markedly different masses.

The changes in gravitational forces related to lunar distance are subtle but are within the same order of magnitude as those associated with the basic electromagnetic properties of the cell membrane that determine the function of the cell. The calculated interaction between gravitational force and magnetic fields produced an electric dipole moment $(\mathrm{A} \cdot \mathrm{m})$. The specific values that are actually measured from membranes and which vary as a function of lunar distance could accommodate unexplained experimental results regarding the interaction between gravitation forces and magnetic fields in biological systems.

\section{References}

[1] C. P. Richter, Biological Clocks in Medicine and Psychiatry, C. C. Thomas, Illinois, 1965.

[2] F. A. Brown, Jr., C. S. Chow, The Biological Bulletin 144 (1973) 437-461.

[3] F. A. Brown, Jr., C. S. Chow, Physiological Zoology 48 (1975) 168-176. 
[4] T. Borowski, International Letters of Chemistry, Physics and Astronomy 1 (2012) 1-5.

[5] M. A. Persinger, The Open Astronomy Journal 5 (2012) 41-43.

[6] M. A. Persinger, Current Medicinal Chemistry 17 (2010) 3094-3098.

[7] P. Andersen, S. A. Andersson, Physiological bases of the alpha rhythm, AppletonCentury Croft: N.Y. 1968.

[8] E. R. Kandel, J. H. Schwartz, T. M. Jessell, Principles of Neural Science, Appleton and Lange, Conn., 1991.

[9] N. A. Aladjalova, Slow electrical processes in the brain, Elsevier: Amsterdam, 1964.

[10] M. A. Persinger, Natural Hazards 2013; DOI 10.1007/s 11069-013-0827-3.

[11] T. Borowski, International Letters of Chemistry, Physics and Astronomy 11 (2013) 44-53.

[12] M. O. Jensen, V. Jogini, D. W. Borhani, A. E. Leffler, R. O. Dror, D. E. Shaw, Science 336 (2012) 229-233.

[13] J. D. Clements, Trends in the Neurosciences 19 (1996) 163-170.

[14] B. T. Dotta, K. S. Saroka, M. A. Persinger, Neuroscience Letters 513 (2012) 151-154.

[15] T. A. Moraes, P. W. Barlow, E. Klingele, C. M. Gallup, Naturwissenschaften 99 (2012) 465-472.

[16] M. A. Persinger, Psychological Reports 28 (1971) 434-438.

[17] M. Hamalainen, R. Hari, R. J. Ilmuniemi, J. Knuutia, O.V. Lounasmaa, Reviews in Modern Physics 65 (1993) 413-497.

[18] T. S. Park, S. Y. Lee, Neuroimage 35 (2007) 531-538. 